\title{
Value of the time trade off method for measuring utilities in patients with rheumatoid arthritis
}

G J Tijhuis, S J T Jansen, A M Stiggelbout, A H Zwinderman, J M W Hazes, T P M Vliet Vlieland

\begin{abstract}
Objective-To assess the feasibility, reliability, and validity of the time trade off (TTO) in patients with rheumatoid arthritis (RA).

Methods-The TTO was applied in 194 patients with RA with increasing difficulty in performing activities of daily living. The test-retest reliability was determined in 35 of these patients and was calculated by the intraclass correlation coefficient (ICC). Construct validity was evaluated with the following sets of variables: measures of utility (rating scale), quality of life (RAND 36 item Health Status Survey (RAND-36) and RAQoL), functional status (Health Assessment Questionnaire, grip strength, and walk test), and disease activity (doctor's global assessment, disease activity score, pain, and morning stiffness).
\end{abstract}

Results-Ten patients (5\%) did not complete the TTO. The median value of the TTO was 0.77 (range $0.03-1.0$ ). The testretest ICC of the TTO was $0.85(\mathrm{p}<0.001)$. Construct validity testing of the TTO showed poor to moderate correlations (Spearman's $r_{\mathrm{s}}$ between 0.19 and 0.36 , $\mathbf{p}<0.01$ ) with all outcome measures except for the subscale role limitation (physical problem) of the RAND-36, the walk test, the doctor's global assessment of disease activity, and morning stiffness. Multiple regression analysis showed that only $17 \%$ of the variance of the TTO scores could be explained.

Conclusions-The TTO method appeared to be feasible and reliable in patients with RA. The poor to moderate correlations of the TTO with measures of quality of life, functional ability, and disease activity suggest that the TTO considers additional attributes of health status. This may have implications for the application of the TTO in clinical trials in patients with RA. (Ann Rheum Dis 2000;59:892-897)

Medical Statistics, Leiden University

Medical Centre

A $\mathrm{H}$ Zwinderman

Correspondence to Dr G J Tijhuis, Leiden University Medical Centre, Department of Rheumatology, C4-R, PO Box 9600, 2300 RC Leiden, The Netherlands

Email:

gjtijhuis@rheumatology.azl.nl

Accepted for publication 10 April 2000

Rheumatoid arthritis (RA) is a chronic disease, which is associated with considerable physical impairment, work disability, and frequent healthcare utilisation. ${ }^{1}$ The continuing development of new treatment strategies for patients with RA and the increasing scarcity of resources make it necessary to prove that treatment strategies are not only effective but also that they are cost effective. Therefore, a growing number of clinical studies in RA include economic evaluations. One method of eco- nomic evaluation is the cost-utility analysis. In this method a utility is used as a global, health related, quality of life measure. A utility is defined as the level of subjective satisfaction, distress, or desirability that people associate with a particular outcome. ${ }^{2}$ It is expressed as a single value on a scale ranging from 0 (usually death) to 1 (usually perfect health). The values that subjects attach to these health states are called the utilities of health states. They are used as quality adjustment factors. Until now, there has been little information about the meaning of utility values in relation to more common outcome measures in patients with RA.

The three most widely used methods of utility measurement are the standard gamble (SG), the time trade off (TTO), and the rating scale (RS) or visual analogue scale (VAS). With the SG, the respondent is asked to make a choice between two options. The first option is the certainty of living for the rest of one's life in a particular health condition. The other option is a gamble with two possible outcomes, living for the rest of one's life in perfect health or immediate death. The chances in the gamble are varied to determine the point at which a respondent is indifferent about the choice between the certain option and the gamble. ${ }^{3}$ The TTO asks the respondent to value health states in terms of duration of life in a state of perfect health that would be equivalent to some period in a particular health condition-for example, the patient's own health state. ${ }^{4}$ In contrast with the SG, in the TTO no element of uncertainty is involved. The RS does not reflect any trade off that a patient may be willing to make in order to obtain better health, either in terms of risk or in life years. ${ }^{5}$ Utilities derived by the TTO and SG are more closely linked to the theoretical foundations of utility measurement than is the case with the RS. ${ }^{6}$ However, it was found that the SG is less responsive in patients with chronic musculoskeletal pain. ${ }^{7}$ Moreover, the TTO has the advantage of being easier to understand and taking less time to administer than the SG. ${ }^{8}$

The TTO has been used in some studies of patients with rheumatic diseases. ${ }^{9-13}$ However, there are no studies in which the feasibility, reliability, and validity of this instrument have been described in patients with RA. Therefore, the aim of this study was to assess these properties in a group of patients with RA.

Patients and methods

PATIENTS

Data were gathered as part of a randomised controlled trial, in which the cost effectiveness 
of three types of care (inpatient multidisciplinary care, multidisciplinary day care, and outpatient care coordinated by a rheumatologist and a nurse practitioner) for patients with RA was compared. The patients were recruited between December 1996 and January 1999 in the Leiden University Medical Centre and five non-academic hospitals within the region of Leiden. Two hundred and ten consecutive patients who visited the outpatient clinic of the rheumatology departments of these hospitals, and who satisfied the inclusion criteria, were included. The inclusion criteria were RA as defined by the 1987 revised ARA criteria ${ }^{14}$ and increasing difficulty in performing activities of daily living for at least six weeks. Functional problems were not solvable by the rheumatologist alone or by a single health professional. Patients who had medical complications of RA requiring immediate admission to hospital and patients who were unable to reach the hospital before 10 am were excluded. The medical ethics committees of all participating hospitals approved the study protocol. All patients gave written informed consent.

METHODS

Two trial nurses who were blinded to the patient's treatment status carried out all assessments. Assessments were made at baseline and weeks $6,12,26,52,78$, and 104 . For the utility study, only the data gathered at baseline were used.

Sociodemographic characteristics and clinical data The following data were recorded at baseline: sex, age, disease duration, rheumatoid factor, status of living (living alone or with partner), and level of education (categorised as low: up to and including lower technical and vocational training; medium: up to and including secondary technical and vocational training; high: up to and including higher technical and vocational training and university).

Utility measures

Two measures of utility were used-the TTO and the RS. The TTO is a method in which the patients were asked how many years $(\mathrm{x})$ in perfect health they would consider equivalent to their remaining life expectancy (y) in the current health state. The number of years in perfect health is varied and the point at which the patient is indifferent about the choice between his own health state for the remaining life expectancy and perfect health for a shorter period of time was used to calculate the utility score. The utility was then calculated as $\mathrm{x} / \mathrm{y}$. A standardised interview format was used, in which the patient was shown a card with a description of perfect health (physical: no complaints or difficulties in performing activities of daily living; psychological: always good and positive feelings and mood; social: all sort of social activities can be performed and contacts with other people are good). To determine the point of indifference between his own health state for the remaining life expectancy and perfect health for a shorter period of time, a visual aid was used. To determine the life expectancy of a patient, the age-specific life expectancy from the general population was used (Netherlands Central Bureau for Statistics, 1995). If it was not possible to complete the method, the patients were asked for the reason. To investigate the test-retest reliability, in 35 consecutive patients who were randomly allocated to receive the inpatient or day care, the TTO method was applied again two weeks after baseline assessments. Both the baseline and the retest assessment took place just before the start of the trial intervention and therefore it was expected that the patients' condition was stable between these two times.

The RS is a method in which the patients are asked to place their current health state alongside a $0-100 \mathrm{~mm}$ vertical line anchored by the "worst imaginable health state" (bottom) and perfect health (top).

\section{Quality of life measures}

Two measures of quality of life (QoL) were used, the RAND-36 and the RAQoL. The RAND 36 item Health Survey 1.0 (RAND-36) contains subscales for physical functioning, social functioning, role limitations (physical problem), role limitations (emotional problem), mental health, vitality, pain, and general health perception. Each scale generates a score from 0 to 100 , with a higher score indicating better health. The RAND-36 may be converted to two summary scales: the physical and mental component summary scales. ${ }^{15}$ The RAND-36 includes the same items as those in the MOS-SF 36 and although the scoring procedures are somewhat different, the effects of these on the scores are minimal. ${ }^{16}$ The RA-specific QoL instrument (RAQoL), which consists of 30 items with a yes/no (1/0) response format, measures different areas of life, including moods and emotions, social life, hobbies, every day tasks, personal and social relationships, and physical contact. ${ }^{17}$ The overall score is the sum of the scores of individual items, with a lower score indicating better QoL. The RAQoL was completed by the patient him or herself. The RAND-36 and the RAQoL have been validated for use in the Netherlands. ${ }^{17}{ }^{18}$

\section{Functional measures}

Functional ability was measured by (a) the Dutch version of the Health Assessment Questionnaire (HAQ) ${ }^{19}(b)$ the grip strength (mean grip strength of the right and the left hand)(Accoson vigorimeter), and (c) the walk test (time needed to walk 50 feet $(15 \mathrm{~m})) .^{20}$

\section{Measures of disease activity}

Measures of disease activity included (a) the patient's global assessment of disease activity, pain, and morning stiffness, all three measured on a horizontal visual analogue scale (VAS, range $0-10 \mathrm{~cm}$ ). The left anchors were no disease activity, no pain, and no stiffness, respectively, and the right, worst imaginable disease activity, severe pain, and severe stiffness, respectively. (b) The doctor's estimation of disease activity, measured on a VAS (range $0-10 \mathrm{~cm}$; anchored on the left by no disease 
Table 1 Characteristics of 194 patients with rheumatoid arthritis

\begin{tabular}{ll}
\hline & Median (range) \\
\hline Age (years) & $58(22-85)$ \\
Duration of disease (years) & $1.84(0.02-46.55)$ \\
& No of patients (\%) \\
Female & $143(74)$ \\
With positive rheumatoid factor & $128(66)$ \\
Living alone & $53(27)$ \\
Education level & \\
Low & $104(54)$ \\
Medium & $65(33)$ \\
High & $25(13)$
\end{tabular}

${ }^{\star}$ Low $=$ up to and including lower technical and vocational training; medium = up to and including secondary technical and vocational training; high $=$ up to and including higher technical and vocational training and university.

activity and on the right by severe disease activity). (c) The number of swollen joints $(0=$ no swelling and $1=$ swelling) using the 28 joint count. $^{21}(d)$ The number of tender joints, including the same joints $(0=$ no pain, $1=$ painful). (e) Erythrocyte sedimentation rate (ESR). From the patient's global assessment of disease activity (VAS), the swollen and tender joint counts, and the ESR, the disease activity score (DAS) was calculated by the following formula: 0.555 (tender joints) +0.284 (swollen joints $)+0.7 \ln ($ ESR $)+0.0142$ (VAS patient's global assessment of disease activity) ${ }^{22}$

\section{Analysis and statistical methods}

To investigate if there were differences in patient characteristics (sex, age, disease duration, level of education) between the group of patients who did and did not complete the TTO method, the Mann-Whitney U test or $\chi^{2}$ test was used where appropriate.

The reliability of the TTO was quantified by the intraclass correlation coefficient (ICC) within the 35 patients in whom the TTO method was applied again after two weeks.

Table 2 Median values and interquartile ranges of measures of utilities, quality of life, functional status, and disease activity in 194 patients with rheumatoid arthritis

\begin{tabular}{|c|c|c|}
\hline & Median & Interquartile ranges \\
\hline \multicolumn{3}{|l|}{ Utility } \\
\hline TTO† $(0-1)^{\star}$ & 0.77 & $0.65-0.91$ \\
\hline Rating scale $(0-100) / 100$ & 0.60 & $0.45-0.70$ \\
\hline \multicolumn{3}{|l|}{ Quality of life } \\
\hline \multicolumn{3}{|l|}{ RAND-36 (0-100): } \\
\hline Physical functioning & 35 & $20-60$ \\
\hline Social functioning & 63 & $38-81$ \\
\hline Role limitations physical functioning & 0 & $0-25$ \\
\hline Role limitations emotional problems & 33 & $0-100$ \\
\hline Mental health & 64 & $52-76$ \\
\hline Vitality & 45 & $35-55$ \\
\hline Pain & 45 & $22-56$ \\
\hline General health perception & 45 & $35-50$ \\
\hline Summary scale mental & 57 & $35-80$ \\
\hline Summary scale physical & 30 & $18-41$ \\
\hline RAQoL (0-30) & 16 & $11-21$ \\
\hline \multicolumn{3}{|l|}{ Functional status } \\
\hline $\mathrm{HAQ}+(0-3)$ & 1.25 & $0.88-1.75$ \\
\hline Grip strength (mm Hg) & 150 & $111-217$ \\
\hline Walk test $(\mathrm{s})$ & 11 & $9-14$ \\
\hline \multicolumn{3}{|l|}{ Disease activity } \\
\hline Disease activity VAS $\uparrow($ investigator) & 38 & $24-52$ \\
\hline Pain VAS & 54 & $33-70$ \\
\hline Morning stiffness VAS & 55 & $30-74$ \\
\hline DAS + & 5.6 & $4.7-6.4$ \\
\hline
\end{tabular}

${ }^{\star}$ Measured in 184 patients, for all other variables $<1.5 \%$ missing data.

†TTO = time trade off; HAQ = Health Assessment Questionnaire; VAS = visual analogue scale (range $0-100)$, DAS $=$ disease activity score.
To investigate a possible influence of patient characteristics, such as age, sex, status of living, and level of education, on the TTO scores the associations between the TTO and these characteristics were quantified by Spearman's correlation coefficients.

In the general population it has been found ${ }^{3}$ that the relation between measures obtained by the RS method and those obtained by the TTO method can be described by the power function RS $=1-(1-\mathrm{TTO})^{0.62}$. As support for the validity of the TTO method, it was further assessed whether after this transformation the RS scores were similar to the TTO scores. The differences between TTO scores and the transformed RS scores were tested for statistical significance by the Wilcoxon signed ranks test.

Construct validity of the TTO was evaluated by quantifying Spearman's correlation coefficients between the TTO method and measures of utility, QoL, functional status, and disease activity. With 194 observations the power to detect correlation coefficients of 0.2 or higher is 0.80 .

To illustrate the ability of the TTO to discriminate between worse and better health state outcomes, the following measures were divided into two categories ( $<$ median and $>$ median values): the RAND-36 (physical and mental), the RAQoL, the VAS pain, and the DAS scores. The HAQ was divided into four parts according to quartiles. Differences of TTO scores between the two health state categories were tested for statistical significance by the Mann-Whitney $U$ test. The differences between the median values of the TTO scores in the four HAQ categories were analysed by analysis of variance.

To determine the contribution of several sets of variables to the explained variance of the TTO, a multiple regression analysis was performed with the following sets of variables: patient characteristics, measures of QoL, functional status, and disease activity. Firstly, we studied sets of variables separately to reduce the problem of multiple testing and to estimate the roles of different types of variables (method enter). The dependent variable was the TTO. Secondly, within each set of variables, the contribution of individual variables was calculated (stepwise forward). Subsequently, the variables from the different sets of variables that contributed significantly to the explained variance $(p<0.001)$ were again analysed into one final model (stepwise forward).

\section{Results}

PATIENT CHARACTERISTICS

Two hundred and ten patients were included in the randomised clinical trial. For logistic reasons, the TTO method was only used after the 16th patient, and was applied in all the following 194 patients. Table 1 shows the sociodemographic and clinical characteristics of the patients. Table 2 shows the median values of measures of utilities, QoL, functional status, and disease activity. 
Table 3 Spearman's correlation coefficients between the time trade off (TTO) and health status outcomes

\begin{tabular}{|c|c|}
\hline & TTO \\
\hline \multicolumn{2}{|l|}{ Utility } \\
\hline TTO $(0-1)$ & - \\
\hline Rating scale $(0-100)$ & $0.36^{\star \star}$ \\
\hline \multicolumn{2}{|l|}{ Quality of life } \\
\hline \multicolumn{2}{|l|}{ RAND-36 (0-100) } \\
\hline Physical functioning & $0.18^{\star}$ \\
\hline Social functioning & $0.25^{\star \star}$ \\
\hline Role limitations physical functioning & 0.09 \\
\hline Role limitations emotional problems & $0.19^{\star \star}$ \\
\hline Mental health & $0.27^{\star \star}$ \\
\hline Vitality & $0.38^{\star \star}$ \\
\hline Pain & $0.22^{\star \star}$ \\
\hline General health perception & $0.18^{\star}$ \\
\hline Summary scale mental & $0.28^{\star \star}$ \\
\hline Summary scale physical & $0.22^{\star \star}$ \\
\hline RAQoL (0-30) & $-0.34^{\star \star}$ \\
\hline \multicolumn{2}{|l|}{ Functional status } \\
\hline $\mathrm{HAQ} \dagger$ & $-0.29^{\star} \star$ \\
\hline Grip strength & $0.26^{\star \star}$ \\
\hline Walk test & -0.07 \\
\hline \multicolumn{2}{|l|}{ Disease activity } \\
\hline Disease activity VAS + (investigator) & -0.09 \\
\hline Pain VAS & $-0.26^{\star \star}$ \\
\hline Morning stiffness VAS & -0.10 \\
\hline DAS $\dagger$ & $-0.19^{\star}$ \\
\hline
\end{tabular}

${ }^{\star} \mathrm{p}<0.05 ;{ }^{\star \star} \mathrm{p}<0.01$

†HAQ = Heath Assessment Questionnaire; VAS = visual analogue scale; DAS = disease activity score.

FEASIBILITY

Ten of the 194 patients (5\%) did not complete the TTO method. Four patients thought the method was too confronting, three patients could not imagine a situation of perfect health, and three patients mentioned religious reasons. There were no significant differences in age, disease duration, education level, and sex between those who completed and those who did not complete the TTO method. However, there was a tendency for higher age (median 65 years) and longer duration of disease (median 2.6 years) in the group of patients who did not complete the TTO than in the group who did complete it (median age 58 years and duration of disease 1.7 years, respectively). Completion of the TTO method took less than 10 minutes.

\section{RELIABILITY}

The two week test-retest reliability was assessed in 35 patients. The ICC for the comparison of both TTO scores was 0.85 $(\mathrm{p}<0.001)$.

\section{VALIDITY}

Associations between utilities and patient

characteristics

No statistically significant correlations were found between the TTO and the patient characteristics (age, sex, status of living, level of education) (data not shown).

\section{Transformation of RS into TTO}

The scores derived by the TTO method were significantly higher than those derived by the RS method $(p<0.001)$. The median value of the transformed RS scores, using the power function $\mathrm{RS}=1-(1-\mathrm{TTO})^{0.62}$, was 0.77 (range 0.3-1.00). There was no significant difference between the median of transformed RS
Table 4 Median scores (range) of the time trade off (TTO) in groups of patients with worse and better health states

\begin{tabular}{|c|c|c|}
\hline & \multicolumn{2}{|l|}{ TTO scores } \\
\hline & $\begin{array}{l}\text { Worse health } \\
\text { statust }\end{array}$ & Better health status $\neq$ \\
\hline $\begin{array}{l}\text { RAND-36 mental } \\
\text { RAND-36 physical } \\
\text { RAQoL } \\
\text { Pain VAS } \\
\text { DAS }\end{array}$ & $\begin{array}{l}0.69(0.03-1.00) \\
0.75(0.03-1.00) \\
0.67(0.03-1.00) \\
0.69(0.03-1.00) \\
0.70(0.03-1.00)\end{array}$ & $\begin{array}{l}0.80^{\star}(0.05-1.00) \\
0.78(0.07-1.00) \\
0.80^{\star \star}(0.31-1.00) \\
0.83^{\star \star}(0.07-1.00) \\
0.80^{\star}(0.04-1.00)\end{array}$ \\
\hline \multicolumn{3}{|c|}{$\begin{array}{l}{ }^{\star} \mathrm{p}<0.01,{ }^{\star \star} \mathrm{p}<0.001 \text {, differences between worse and better } \\
\text { health status (Mann Whitney U test). } \\
\text { +Worse health status and } \ddagger \text { better health status corresponds with } \\
\text { less than or greater than the median value of the outcome meas- } \\
\text { ure; the median values of the outcome measures were RAND-36 } \\
\text { mental 50; RAND-36 physical 50; RAQoL 16; Pain VAS } 50 \text {; } \\
\text { DAS 5.6. } \\
\text { TVAS = visual analogue scale; DAS = disease activity score. }\end{array}$} \\
\hline \multicolumn{3}{|c|}{$\begin{array}{l}\text { Table } 5 \text { Time trade off (TTO) scores within different } \\
\text { categories of the Health Assessment Questionnaire (HAQ) }\end{array}$} \\
\hline$H A Q$ score & \multicolumn{2}{|c|}{ TTO median (range) } \\
\hline $0.00-0.75$ & \multicolumn{2}{|c|}{$0.87(0.30-1.0)$} \\
\hline $0.76-1.50$ & \multicolumn{2}{|c|}{$0.74(0.03-1.0)$} \\
\hline $1.51-2.25$ & \multicolumn{2}{|c|}{$0.67(0.04-1.0)^{\star}$} \\
\hline $2.26-3.00$ & \multicolumn{2}{|c|}{$0.67(0.05-1.0)^{\star}$} \\
\hline
\end{tabular}

${ }^{\star} \mathrm{p}<0.01$ (in comparison with 0.87 ) (analysis of variance).

scores and the median of actual scores of the TTO (median 0.77, range 0.03-1.00) $(\mathrm{p}=0.384)$.

Association between utilities and health status outcome measures

Poor to moderate correlations were found between the TTO scores and the RS, and measures of QoL, functional status, and disease activity (table 3). All correlations between the TTO and the different outcome measures indicated that a better outcome for utilities, QoL, functional status, and disease activity was associated with higher TTO scores. Tables 4 and 5 illustrate the discriminating properties of the TTO for two ("worse and better") categories of the RAND-36, RAQoL, VAS pain, and DAS scores and for four categories of the HAQ, respectively.

Regression analysis

In a multiple regression analysis (method enter) the set of variables of QoL, functional status, and disease activity explained $19 \%$, $10 \%$, and $10 \%$ of the variance of the TTO, respectively. After stepwise forward analysis within each set of variables it appeared that the following variables contributed significantly to the explained variance $(\mathrm{p}<0.001)$ : the RAQoL and the subscale vitality of the RAND-36 (set QoL), the HAQ score (set functional status), and the VAS pain (set disease activity). Stepwise forward analysis of these four variables into one model resulted in a significant contribution of $17 \%$ to the explained variance of the TTO of two variables: the RAQoL and the subscale vitality of the RAND-36 $(p<0.001)$.

\section{Discussion}

The purpose of this study was to assess whether the TTO is a feasible, reliable, and valid instrument to measure utilities in patients 
with RA. The feasibility of applying the TTO method was generally satisfactory. Only 10 (5\%) patients did not complete the TTO method. These patients thought the method was too confronting, or they could not imagine a health status of perfect health, or they would not answer for reasons of a religious nature. The possibility of difficulties of religious nature has been put forward by other authors. ${ }^{23}{ }^{24}$ There was a tendency for older patients with longer disease duration to have difficulty completing the TTO method in comparison with those who did complete it. This age phenomenon has also been found in other patient groups in which the TTO and the SG were applied. ${ }^{25}$ The reliability of the TTO method was good. In other studies of utilities in patients with rheumatic diseases, comparable values of the ICC have been found for the TTO and for other measures of utility. ${ }^{26}$

The median value of 0.77 for the TTO method in this group of patients corresponds with values derived by the same method in other groups of patients with RA,,${ }^{90}$ osteoarthritis, ${ }^{11} 12$ and SLE. ${ }^{13}$

A significant difference was found between scores derived by the TTO method and by the RS. This is consistent with the well known empirical finding that TTO scores are generally higher than RS scores. ${ }^{27}{ }^{28}$ The difference may be explained by the difference in tasks. The TTO requires a trade off, whereas with the RS no consequences about life are linked for the respondent. Therefore, the patients who rate their own health state on a $0-100 \mathrm{~mm}$ line may use this line more equally than is the case with the TTO method. ${ }^{29}$

On the basis of Spearman's correlation coefficient between the TTO and the RS, we can conclude that for individual subjects the RS method is poorly correlated with the TTO method. However, for groups, by using the power function $\mathrm{RS}=1-(1-\mathrm{TTO})^{0.62}$, the median values of the TTO and the transformed RS scores did not significantly differ from each other. This is a finding previously described in other patient groups ${ }^{3}$ and supports the validity of the TTO in this group of patients with rheumatic diseases.

Evaluation of the construct validity showed that the TTO is only poorly to moderately related to measures of QoL, functional ability, and disease activity. The correlation coefficients between the TTO and the RAND-36 correspond with results found in studies with other patient groups in which the MOS-SF 36 and the RAND-36 were used. ${ }^{25}{ }^{30}{ }^{31}$ Regression analysis shows that the combination of the subscale vitality of the RAND-36 and the RAQoL explained only a small part of the variance of the TTO method. The results of the RAND-36 corresponded with those of other studies. $^{32} 33$

The results of the correlations and regression analysis suggest that the TTO is influenced by other factors that may or may not be related to health status. Factors that may possibly contribute to utilities are adaptation to illness, knowledge about the disease, previous experience with disease related problems, and individual beliefs about health in general. Moreover, factors such as family life (partner, children), social networks, and financial status may have a role..$^{34}$ These factors are probably difficult to influence with current interventions.

In conclusion, the TTO method appeared feasible and reliable in a group of patients with RA with increasing difficulty in activities of daily living. Although related to measures of QoL, functional ability, and disease activity it seems that the TTO utilities deal with additional attributes of health status. This may have implications for the application of the TTO in clinical trials, because the sensitivity of the method for detecting clinically relevant differences may be limited. Further research into the usefulness of the method in different groups of patients with RA and in RA clinical trials, including cost-utility analysis, is warranted.

We thank the following rheumatologists who participated in the study: JM de Jonge-Bok, Groene Hart Ziekenhuis, Gouda; Dr C Mallee, Kennemer Gasthuis, Haarlem and JPh Terwiel, Spaarne Ziekenhuis, Haarlem; Dr AJ Peeters and Dr D van Zeben, Reinier de Graaf Gasthuis, Delft; JAPM Ewals, Rode Kruis Ziekenhuis, Den Haag; GA Kok and Dr HK Ronday, Leyenburg Ziekenhuis, Den Haag; I Speyer and Dr ML Westedt, Bronovo Ziekenhuis, Den Haag. In addition, we are indebted to all rheumatology nurse specialists from the participating hospitals. We would like to acknowledge I Henkes and B Oud for making the assessments and $M$ de Best-Bosz, H Ravensbergen-van de Berg, and A van der Blom for secretarial assistance.

This study was financially supported by Het Nationaal Reuma Fonds, The Dutch Arthritis Association, Grant No 931.

1 Pincus T, Callahan LF. The 'side effects' of rheumatoid arthritis: joint destruction, disability and early mortality. $\mathrm{Br}$ Rheumatol 1993;32(suppl 1):28-37.

2 Bush JW. Relative preference versus frequencies in healthrelated quality of life evaluations. In: Wenger N, Mattson ME, Furberg CD, Elison J, eds. Assessment of quality of life in clinical trials of cardiovascular therapies. New York: Le Jacq, 1984:118-39.

3 Torrance GW. Social preferences for health states: an empirical evaluation of three measurement techniques. Socio-econ Planning Sci 1976;10:129-36.

4 Torrance GW, Thomas WH, Sackett DL. A utility maximization model for evaluation of health care programs. Health Zerv Res 1972;7:118-33.

5 Robinson A, Dolan P, Williams A. Valuing health status using VAS and TTO: what lies behind the numbers? Soc Sci Med 1997;45:1289-97.

6 von Neumann J, Morgenstern O. Theory of games and economic behaviour. Princeceton: Princeton University Press, 1947

7 Goossens MEJB, Vlaeyen JWS, Rutten-van Mölken MPMH, van der Linden SMJP. Patient utilities in chronic musculoskeletal pain: how useful is the standard gamble method? Pain 1999;80:365-75.

8 Russell LB, Gold MR, Siegel JE, Daniels N, Weinstein MC. The role of cost-effectiveness analysis in health and medicine. Panel on Cost-Effectiveness in Health and Medicine. JAMA 1996;276:1172-7.

9 Ferraz MB, Quaresma MR, Goldsmith CH, Bennett K, Atra E. Estimation of benefits and risks of the treatment of rheumatoid polyarthritis with glucocorticoids using the health-related quality of life measurements. Rev Rhum Ed Fr 1994;61:255-9.

10 Lambert CM, Hurst NP, Forbes JF, Lochhead A, Macleod $M$, Nuki $G$. Is day care equivalent to inpatient care for active rheumatoid arthritis? Randomised controlled clinical active rheumatoid arthritis? Randomised controlle

11 Laupacis A, Bourne R, Rorabeck C, Feeny D, Wong C, Tugwell $\mathrm{P}$, et al. The effect of elective total hip replacement on health-related quality of life. J Bone Joint Surg Am 1993;75:1619-26

12 Katz JN, Phillips CB, Fossel AH, Liang MH. Stability and responsiveness of utility measures. Med Care 1994;32: $183-8$.

13 Moore AD, Clarke AE, Danoff DS, Joseph L, Bélisle P, Neville C, et al. Can health utility measures be used in lupus research? A comparative validation and reliability study of 4 utility indices. J Rheumatol 1999:26:1285-90.

14 Arnett FC, Edworthy SM, Bloch DA, McShane DJ, Fries JF, Cooper NS, et al. The American Rheumatism Association 1987 revised criteria for the classification of rheumatoid arthritis. Arthritis Rheum 1988;31:315-24.

15 Ware JE Sherbourne CD. The RAND-36 Short-form Health Status Survey: 1. Conceptual frame work and item selection. Med Care 1992;30:473-81. 
16 Hays RD, Sherbourne CD, Mazel RM. The RAND 36-Item Health Survey 1.0. Health Econ 1993;2:217-27.

17 De Jong Z, van der Heijde D, McKenna SP, Whalley D. The reliability and construct validity of the RAQoL: a rheumatoid arthritis-specific quality of life instrument. Br J Rheumatol 1997;36:878-83.

18 Van de Zee KI, Sanderman R. Het meten van de algemene gezondheidstoestand met de RAND-36. Groningen: Rijksuniversiteit, 1993. (ISBN 90-72156-60-9.)

19 Siegert CE, Vleming LJ, Vandenbroucke JP, Cats A. Measurement of disability in Dutch rheumatoid arthritis patients. Clin Rheumatol 1984;3:305-9.

20 Grace EM, Gerecz EM, Kassam YB, Buchanan HM, Buchanan WW, Tugwell PS. 50-foot walking time: a critical assessment of an outcome measure in clinical therapeutic trials of antirheumatic drugs. Br J Rheumatol 1988;27: $372-4$.

21 Smolen JS, Breedveld FC, Eberl G, Jones I, Leeming M, Wylie GL, et al. Validity and reliability of the twenty-eightjoint count for the assessment of rheumatoid arthritis activity. Arthritis Rheum 1995;38:38-43.

22 Prevoo MLL, van 't Hof MA, Kuper HH, van Leeuwen MA, van de Putte LBA, van Riel PLCM. Modified disease activity scores that include twenty-eight joint counts. Development and validation. Arthritis Rheum 1995;38: 44-8.

23 Bursztajn H, Hamm RM. The clinical utility of utility assessment. Med Decis Making 1982;2:161-5.

24 Stiggelbout AM, Kiebert GM, Kievit J, Leer JWH, Habbema JDF, De Haes JCJM. The 'utility' of the time trade-off method in cancer patients: feasibility and proportional trade-off. J Clin Epidemiol 1995;48:120714.
25 Bosch JL, Hunink MG. The relationship between descriptive and valuational quality-of-life measures in patients with interm $16: 217-25$

26 Bakker C, Rutten-van Mölken M, Hidding A, van Doorslaer $\mathrm{E}$, Bennett K, van der Linden S. Patient utilities in ankylosing spondylitis and the association with other outcome

measures. J Rheumatol $1994 ; 21: 1298-304$.
27 Torrance GW. Utility approach to measuring health-related quality of life. Journal of Chronic Disease 1987;40:593603.

28 Torrance GW, Feeny D. Utilities and quality-adjusted life years. Int J Technol Assess Health Care 1989;5:559-75.

29 Read JL, Quinn RJ, Berwick DM, Fineberg HV, Weinstein MC. Preferences for health outcomes. Comparison of assessment methods. Med Decis Making 1984;4:315-29.

30 Revicki DA, Kaplan RM. Relationship between psychometric and utility-based approaches to the measurement of ric and utility-based approaches to the measurement of
health-related quality of life. Qual Life Res 1993;2:477-87.

31 Blumenschein K, Johannesson M. Relationship between quality of life instruments, health state utilities, and quality of life instruments, health state utilities, and
willingness to pay in patients with asthma. Ann Allergy willingness to pay in patients with

32 Fryback DG, Dasbach ED, Klein R, Klein BEK, Martin PA, Dorn N, et al. Health assessment by SF-36, quality of wellbeing index, and time tradeoffs: predicting one measure from another. Med Decis Making 1992;12:348.

33 Tsevat J, Solzan JG, Tolan KK, Soukup JR, Cook EF, Tosteson ANA, et al. Health status and utilities of HIV+ and primary care patients [abstract]. Clinical Research 1992;40: 569A.

34 Chapman GB, Elstein AS, Kuzel TM, Sharifi R, Nadler RB, Andrews A, et al. Prostate cancer patients' utilities for health states: how it looks depends on where you stand. Med Decis Making 1998;18:278-86. 